\title{
Téoros
}

Revue de recherche en tourisme

\section{Jeunes femmes en voyage}

Une expérience tunisienne

\section{Stéphanie Laporte}

Volume 22, numéro 1, printemps 2003

Tourisme et sexualité

URI : https://id.erudit.org/iderudit/1071582ar

DOI : https://doi.org/10.7202/1071582ar

Aller au sommaire du numéro

Éditeur(s)

Université du Québec à Montréal

ISSN

0712-8657 (imprimé)

1923-2705 (numérique)

Découvrir la revue

\section{Citer cet article}

Laporte, S. (2003). Jeunes femmes en voyage : une expérience tunisienne.

Téoros, 22(1), 29-35. https://doi.org/10.7202/1071582ar

Ce document est protégé par la loi sur le droit d'auteur. L'utilisation des services d'Érudit (y compris la reproduction) est assujettie à sa politique d'utilisation que vous pouvez consulter en ligne.

https://apropos.erudit.org/fr/usagers/politique-dutilisation/
Cet article est diffusé et préservé par Érudit.

Érudit est un consortium interuniversitaire sans but lucratif composé de l'Université de Montréal, l'Université Laval et l'Université du Québec à Montréal. Il a pour mission la promotion et la valorisation de la recherche. https://www.erudit.org/fr/ 


\section{Jeunes femmes en voyage}

\section{Une expérience tunisienne}

\section{Stéphanie Laporte}

Nadhraton, fa basmaton, fa hamsaton, fa salamon, fa kalamon, fa maïoyidon, fa liqaa.

Un regard, une esquisse de sourire, un chuchotement, un bonjour, une discussion, un rendez-vous, une rencontre.

Proverbe arabe

\section{Introduction}

e tourisme et les voyages constituent l'une des dimensions saillantes de la culture occidentale contemporaine, contribuant de façon significative au développement économique et social des pays qui offrent des destinations touristiques. Si de nombreuses études ont été consacrées au tourisme d'un point de vue masculin et masculiniste (Johnston, 2001), les caractéristiques des femmes touristes et leurs expériences, quant à elles, ont été moins bien cernées. Pourtant, le tourisme féminin est en augmentation constante et s'accompagne du développement de services de voyage spécialisés, que ce soit sous la forme de revues féminines spécialisées, de sites Internet, de forums d'échanges, de destinations spécifiques, de tours organisés ou de clubs de voyage.

Les études contemporaines consacrées aux touristes occidentales ont surtout porté sur l'analyse de leurs voyages dans les pays du Sud et sur leur quête d'expériences sexuelles ou romantiques, que ce soit en Jamaïque (De Albuquerque, 1998 ; Pruitt et Lafont,
1995), aux Barbades (De Albuquerque, 1998 ; Karch et Dann, 1981), en Équateur (Meisch, 1995) ou en République dominicaine (Herold, Garcia et DeMoya, 2001), et elles ont mis en relief leurs modalités de séduction et de rencontres sexuelles. Cependant, ces études présentaient généralement le point de vue des hommes qui entraient en relation avec des voyageuses, ignorant ainsi les motivations et les expériences spécifiques des femmes. De plus, quand elles se limitent au tourisme sexuel, les recherches ne permettent pas de saisir la diversité des motivations et des expériences de voyage des femmes, comme le suggèrent les travaux de Netzley (2001) et de McGehee, Loker-Murphy et Uysal (1996). Dans une telle perspective, la présente étude exploratoire vise à dégager les expériences touristiques de neuf étudiantes québécoises, célibataires, âgées en moyenne de vingtdeux ans, qui ont effectué un voyage de onze jours en Tunisie, et de saisir leur inscription en tant que femmes dans ce contexte socioculturel à la fois marqué par la tradition musulmane et ouvert sur un tourisme international important. Celui-ci est favorisé par la proximité de l'Europe, par son climat tempéré et par ses ressources culturelles et environnementales originales (Smaoui, 1992). Le tourisme constitue ainsi l'un des secteurs clés de l'économie de la Tunisie, pays de plus en plus populaire auprès des vacanciers ; autour de quatre millions de visiteurs dans les dernières années - dont au moins 15000 Canadiens, $85 \%$ de Québécois - y ont séjourné pendant treize jours en moyenne (La Presse, 2002).

Nous avons utilisé plusieurs approches pour dégager les perceptions et les expériences des participantes dans le contexte de ce voyage, dont la méthode de l'observation-participante, l'auteure de ce texte ayant participé au voyage. Pendant le voyage de retour en avion, l'observatrice a également demandé à toutes les participantes de noter les expériences importantes qu'elles avaient vécues dans le but de constituer un aide-mémoire qui servirait aux entrevues ultérieures. Deux étudiantes avaient par ailleurs tenu un journal de bord durant le voyage. Par la suite, les entrevues semi-structurées ont couvert quatre grands thèmes : 1) motivations du voyage et représentations de la Tunisie ; 2) représentations du monde masculin ; 3) représentations du monde féminin ; et 4) retour sur le voyage. Les entrevues ont été codifiées puis comparées selon les différentes catégories d'analyse afin de dégager les convergences et les écarts dans les expériences.

\section{Motivations du voyage et représentations de la Tunisie}

Plusieurs motivations combinées se retrouvent dans la décision des étudiantes de voyager en Tunisie : la participation à

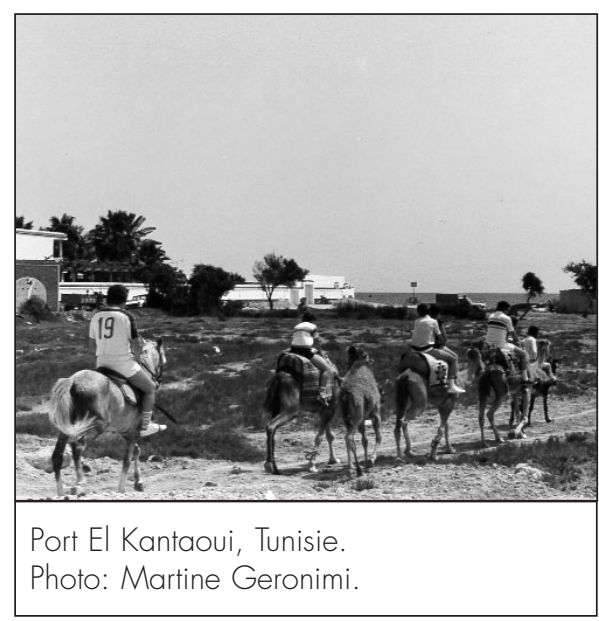



jectif essentiel du voyage, ce qui leur permettait d'approfondir leur formation professionnelle et scientifique, mais la connaissance des aspects culturels et touristiques du pays était avancée comme motif. Quelques étudiantes ont abordé la question des dimensions romantiques, mais aucune n'a insisté sur une finalité sexuelle directe :

On faisait des farces, avant de partir, sur le fait de rencontrer un beau viendrait jamais, comme dans les romans Harlequin. Mais, en fait, on ne savait même pas si c'était des beaux gars, on ne savait pas de quoi ils avaient l'air. On savait qu'ils avaient le teint foncé un peu et que ça nous plaisait bien.

Tout comme dans la recherche de De Kadt (1979) qui indiquait que la plupart des touristes visitant la Tunisie ignoraient les caractéristiques de la société et de la culture de ce pays, la grande majorité des étudiantes avait, avant le voyage, une image assez floue de leur destination et des caractéristiques géographiques, sociales et culturelles de la Tunisie. Leur information provenait de sources comme la télévision, la presse ou un congrès scientifique constituait l'obmédecin tunisien et qu'on ne re-

des personnes de leur entourage qui avaient déjà visité le pays. Peu d'étudiantes avaient eu recours à des lectures avant le départ pour mieux connaître la Tunisie.

La plupart étaient au courant que c'était un pays musulman d'Afrique du Nord, influencé par l'Europe et la France, et qu'il se représentait comme plus paisible que les autres pays du Maghreb ; un beau pays, marqué par la mer et le désert. Plusieurs ont insisté sur les différences de mentalité avec le Québec, en particulier dans les rapports hommes-femmes marqués par le machisme et le statut problématique des femmes, qu'elles associaient quelquefois à la montée de l'intégrisme. Les avis divergeaient quant aux règles à suivre dans les rapports avec les hommes. Certaines avaient reçu le conseil de toujours porter une tenue vestimentaire modeste et de demeurer prudentes dans leurs rapports avec les hommes, alors que d'autres avaient été informées que les hommes étaient charmants et habitués à rencontrer des touristes. Une session d'information avec l'organisateur tunisien du voyage, avant le départ, a apporté des précisions sur l'histoire socioculturelle du pays et l'itinéraire prévu, ce qui a contribué à atténuer chez quelques étudiantes certaines anxiétés associées au voyage, alors que plusieurs autres restaient plutôt

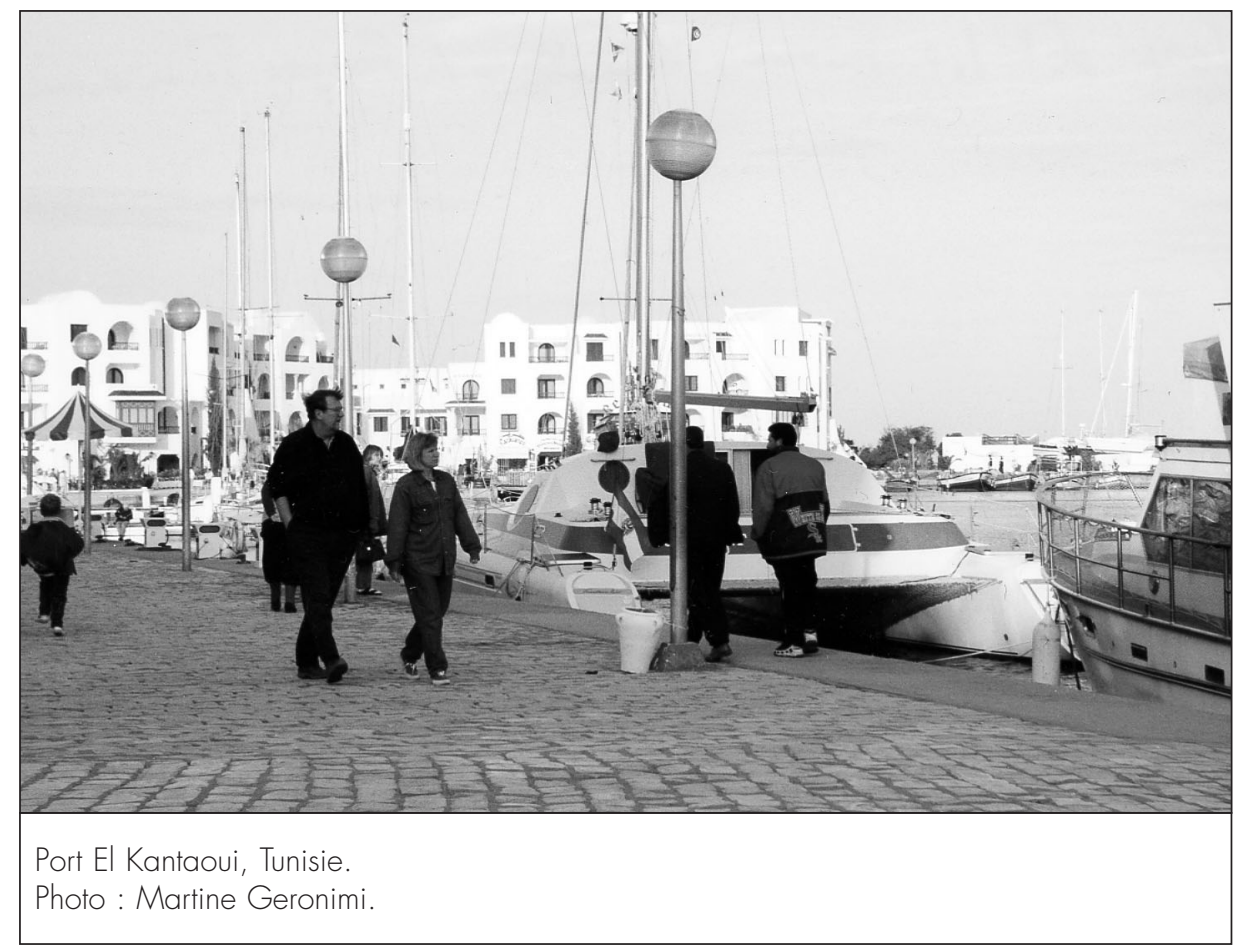

craintives, ne sachant pas à quoi s'attendre. Le voyage leur a permis d'entrer en contact avec la diversité des milieux de vie tunisiens, la grande ville, Tunis, une destination touristique comme Hammamet et les villes et oasis du sud, ce qui a donné lieu à des expériences multiples et contrastées des mondes masculin et féminin.

\section{Représentations du monde masculin}

Une fois en Tunisie, l'une des préoccupations quotidiennes a tourné autour du type de vêtements appropriés que les étudiantes devaient porter. Comme le voyage s'est fait pendant le mois de mai, période où la chaleur est déjà élevée, le choix des vêtements de type estival (shorts, camisoles, robes légères) s'est posé avec acuité dans la mesure où les Tunisiennes respectent les règles de modestie vestimentaire, sans pourtant renoncer aux vêtements européens. La plupart des étudiantes, prétextant la chaleur, le confort, leur statut d'étrangère et de touriste, ne se considéraient pas comme obligées de se conformer aux règles vestimentaires du pays, une décision renforcée par les commentaires des Tunisiens rencontrés qui leur suggéraient de maintenir leurs habitudes, même si certaines étaient conscientes des réactions stigmatisantes - associées à la prostitution - que leur tenue pouvait provoquer. D'autres, par contre, se sont ralliées à une modestie vestimentaire après avoir pris conscience des réactions que leur tenue provoquait. D'autres, enfin, ont surveillé davantage leur tenue pour ne pas attirer les regards et respecter la culture tunisienne.

Presque toutes les étudiantes étaient d'accord pour dire que l'habillement a joué un rôle dans les réactions et les attitudes des hommes envers elles. Les questions du regard et de la proximité physique constituent un thème saillant dans les entrevues de toutes les étudiantes et leur importance varie en fonction des catégories d'hommes rencontrés, soit dans des cadres formels, des contextes touristiques anonymes or lors de relations plus intimes. Dans le cadre des relations sociales associées au congrès, où se trouvaient des Occidentaux et des Tunisiens occidentalisés, le type d'interactions obéissait aux critères 
occidentaux, marqués par une certaine distance physique et une certaine neutralité du regard, ce que Goffman (1966) nomme l' « inattention courtoise », dans la mesure où le regard empreint d'une certaine affectivité est réservé aux proches.

Soulignons que la façon de regarder des Américains et des Européens du Nord est souvent perçue dans la plupart des pays arabes, en Amérique latine et en Europe méridionale comme une marque d'impolitesse, de malhonnêteté ou, pire, d'hypocrisie. Il existe d'ailleurs des différences à l'intérieur même de la culture occidentale, les Américains regardant le plus franchement les étrangers comparativement aux Européens et aux Britanniques. Dans les centres urbains et dans les rapports avec les hommes tunisiens croisés dans la rue et sur les lieux touristiques (hôtels, bars, restaurants, magasins), les règles culturelles associées au regard et aux contacts physiques s'éloignent alors des modèles occidentaux, ce qui n'est pas sans créer des malaises, sinon des tensions interpersonnelles.

Plusieurs étudiantes percevaient alors le regard comme omniprésent et insistant, cherchant constamment le contact visuel. Elles le codifiaient comme une tentative de séduction constante de la part des hommes, mais aussi quelquefois comme une forme de harcèlement, voire même une forme d'agression, lassante à la longue. D'autres acceptaient sans trop de problèmes de se voir constamment dévisagées ou se trouvaient gênées et même coupables de provoquer de telles réactions, modifiant quelquefois leur tenue vestimentaire pour tenter de détourner les regards.

C'est impressionnant de voir que ce ne sont pas seulement des hommes qui nous regardent, mais aussi des enfants, que ce soit dans la rue ou dans les restaurants.

Les hommes ont un regard très profond, très dominateur, malgré les beaux sourires, ça m'intimide énormément. [...] Ça se passe toujours avec les yeux; ils ont un contact énorme avec les yeux, mais tu ne sais jamais quoi déceler. Je les ai trouvés mystérieux. Ils ne se révèlent pas facilement.
On se faisait fixer et il n'y avait pas de gêne à faire cela là-bas, ce n'était pas subtil. Les garçons s'arrêtaient dans la rue et nous fixaient jusqu'à temps que l'on disparaisse. À la fin, je ne trouvais plus cela drôle de me faire regarder et je me suis mise à penser que c'était peut-être ma faute. Ils ne sont pas fous et si on leur offrait quelque chose à regarder, ils regardaient. C'était peut-être nous qui avions provoqué ce jeu.

Un tel malaise révèle la présence d'un choc culturel important associé au passage d'une culture où le contact visuel est secondaire à une autre où le contact visuel est dominant, ce qui peut représenter une source d'anxiété considérable pour des personnes provenant de cultures du premier type (Argyle et Dean, 1965), comme c'est le cas au Québec, transplantées dans une culture du second type, ici la Tunisie. Des études anthropologiques menées sur l'aire culturelle arabo-musulmane ont montré l'importance accordée au regard. Pour Couchard (1994), la vue serait l'organe des sens le plus sacralisé dans l'Islam et plusieurs lois encadrent d'ailleurs ce qui peut être regardé. Ainsi, certaines zones corporelles sont interdites à la vue en fonction du sexe et du statut familial (Maison de l'Islam, 2003).

Le regard a, par ailleurs, une fonction sociale importante. Dans cette aire culturelle, selon les observations de Watson (1970), le fait de ne pas regarder son interlocuteur en face ou de détourner le regard durant une conversation est un signe d'impolitesse, alors que le contact des yeux, au contraire, est un signe de franchise. Watson et Graves (1966) et Watson (1970) ont par ailleurs constaté que des dyades d'étudiants provenant de cultures arabes échangeaient plus fréquemment des regards lors d'une conversation comparativement à des étudiants américains. Chebel, quant à lui, situe l'importance du regard dans le contexte des stratégies de séduction dans le monde islamique, appuyant sur la notion de « consommation scopique ». Ainsi, la sensualité est déjà présente dès le premier échange des regards, « car du regard naît le lien et du lien se cristallise cet amour » (1995 : 34). Dans la culture populaire, la « flamme » qui se dégage des yeux serait le reflet de la vie intérieure. Aussi l'u-

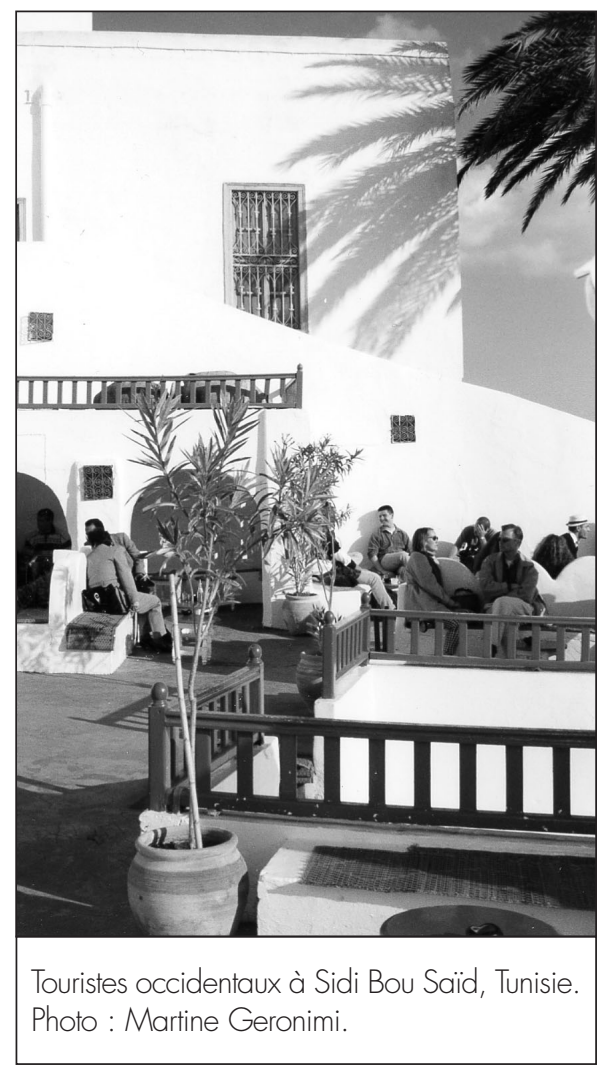

tilisation importante du khôl dans ces civilisations aurait-elle pour but de rehausser la présentation de son soi intérieur. L'œillade, le regard désirant (Chebel, 1995) anciennement réservé aux hommes, mais aujourd'hui utilisé également par les femmes, est particulier dans le processus de séduction puisqu'il permet de se signifier un intérêt mutuel, le contact physique ne pouvant s'établir compte tenu de la séparation relative des sexes. C'est donc dire toute l'importance du regard dans l'établissement de la relation. La primauté du regard ne doit pas cacher cependant des différences aussi significatives dans le champ de la proximité physique et des contacts corporels permis ou non. De ce point de vue, les recherches sur la culture arabo-musulmane montrent que, dans le contexte d'une conversation (Watson, 1970), les individus ont tendance à réduire la distance physique, associée au regard, mais il n'est pas clair si cette proximité est dépendante du sexe des interlocuteurs. En effet, les contacts avec les femmes sont davantage contrôlés sinon généralement interdits dans la sphère publique, alors que ceux entre hommes ne sont pas inhibés. Chebel parle à ce sujet d'une homosensualité, une propension « à reporter sur leurs 
pairs l'excédent de sensualité qu'ils n'arrivent pas à écouler autrement » (ibid. : 312). Cette homosensualité se caractérise par des comportements identifiés, comme se tenir en groupe et se tenir par la main, comportements que certaines des étudiantes ont observés, non sans surprise.

Les hommes s'embrassent, les amis de gars se prennent par le cou, par la taille et ils marchent ensemble, ils se prennent même la main. Le toucher n'est pas vulgaire ou déplacé.

Elles insistent d'ailleurs sur les différences de culture dans ce domaine en faisant remarquer que les rapports d'intimité et les frontières corporelles sont moins rigides qu'au Québec, même si la distance intellectuelle et émotionnelle reste plus grande. Par contre, plusieurs étudiantes ont expérimenté des contacts physiques non voulus (attraper le bras, prendre les mains, toucher les seins) qui étaient considérés comme une forme de harcèlement sexuel contre lequel elles avaient à s'élever, développant des stratégies personnelles ou collectives pour s'en protéger (ne pas regarder les personnes, ne pas parler, repousser les contacts, se déplacer en groupe). Ces gestes qui ont été vécus quelques fois comme une agression, une intrusion dans leur bulle personnelle, et semblent être fréquemment associés aux visites des souks, ou marchés locaux.

On était tellement à l'étroit que les hommes pouvaient nous toucher, nous attraper le bras. On s'est retrouvé dans une espèce de jungle, on se protégeait en se tenant bras dessus bras dessous. C'était continuellement des paroles, des interpellations, des touchers.

Dans le souk même, on se faisait harceler, suivre, toucher, dire des cochonneries. Je n'ai pas aimé ça. Il ne faut pas que tu les regardes car aussitôt que tu les regardes, pour eux le contact est établi; ils se mettent à te parler, à te suivre. Tu es faite.

Certaines se sont senties responsables de tels comportements, les attribuant à la légèreté de leurs vêtements ou à des attitudes provocantes dont elles n'étaient pas conscientes.
Même si tu sais qu'il ne va rien t'arriver, tu deviens oppressée, mal dans ta peau, et tu commences à te dire que c'est toi qui n'es pas à ta place, que c'est toi qui provoques les gens, ou ta tenue. Si on avait été plus habillé, peut-être que ça aurait été différent.

Plus rarement, des gestes à connotation directement sexuelle, comme tirer la langue de façon suggestive, ont été observés. Les étudiantes ont aussi noté le recours à des compliments fréquents, accompagnant le regard, vantant leur beauté et leur grâce de façon stéréotypée.

Le monde te siffle tout le temps : « gazelles, gazelles»!

Ils nous font sentir comme si on était les plus belles filles au monde, ils nous comparent à Céline Dion et nous disent qu'on est les plus belles.

Tu es constamment observée et parfois même admirée parce que ce n'est pas seulement négatif, on reçoit aussi des compliments; mais ça finit quand même par être fatigant à la longue.

Les hommes tentent aussi d'établir des liens en les interrogeant sur leur pays d'origine ou en leur adressant la parole. La plupart des étudiantes étaient enclines à maintenir une distance totale, d'autres s'astreignaient à un minimum de politesse, constatant par ailleurs que ces aspects de l'ethos tunisien étaient absents dans les milieux ruraux, plus enclins à maintenir une distance avec les visiteurs étrangers.

L'établissement de relations plus directes avec des jeunes hommes intéressés à rencontrer ces jeunes touristes a pris plusieurs formes. Quelques étudiantes rapportent avoir établi des relations amicales et polies avec des jeunes hommes qui travaillaient surtout dans les hôtels et qui leur ont fourni des renseignements sur les restaurants locaux ou des aperçus de la culture locale et qui leur faisaient visiter des lieux jugés hors limites pour les touristes, comme les cafés locaux. Ils pouvaient aussi leur servir d'escorte pour entrer dans des clubs ou des bars ou les accompagner dans des excursions en mer :
Nous nous sommes fait des amis làbas. Trois garçons entre autres qui nous ont servi de guide et qui étaient très corrects ; ils nous ont dit n'avoir aucune idée derrière la tête. Nous ne les avons pas revus, donc je ne peux pas dire si la relation aurait changé à la longue.

Je m'étais fait un ami, on a passé une journée ensemble. Puis, il m'a téléphoné le soir. Il était super gentil, il m'avait aidée à magasiner dans les souks. Ce n'est pas moi qui l'ai sollicité. Il était jeune, fin, il était correct, parce que sinon je lui aurais dit «fais de l'air». C'est lui qui voulait nous accompagner, il voulait nous aider. Il m'a demandé si j'avais un chum, j'ai dit «oui »! Il m'a appelée à l'hôtel, on a jasé et puis il m'a souhaité un bon voyage de retour. Pas d'allusions, pas de « cruisage».

Certains hommes ont aussi tenté de développer des relations plus sentimentales, refusées par les étudiantes, surprises de la rapidité des déclarations d'amour et les sentiments de possession et de jalousie à leur égard, ce qui pouvait aller jusqu' au harcèlement et à une surveillance constante finissant par créer des tensions interpersonnelles qui ont nécessité une mise au point abrupte.

J'ai rencontré un gars qui avait l'air bien gentil, bien joli, bien intéressant ; j'ai juste parlé avec pour ce soir-là et je l'ai revu après quelquefois. Il me suivait tout le temps, il était toujours après moi ; il m'a même fait une déclaration d'amour, dit qu'il voulait venir au Québec, qu'il était en amour avec moi. [...] Je me suis rendu compte qu'il était jaloux, possessif ; j'avais beau lui dire que j'avais un copain, ça ne le dérangeait pas ; il ne comprenait pas, il essayait toujours de me tirer par la main, de me guider, de tout faire pour moi ; il m'énervait vraiment.

Les demandes sexuelles ont aussi fait partie du répertoire des stratégies des dragueurs, ou « bezness » (Lévy, Laporte et El Feki, 2001), ou du personnel travaillant directement avec les touristes, comme les 
guides. Les initiatives pouvaient être indirectes, par exemple inviter discrètement une étudiante à une halte au domicile sous prétexte de se reposer, ou plus directes, allant jusqu'à des appels téléphoniques le soir à la chambre d'hôtel pour initier une conversation souvent perçue comme ambiguë, pleine de sous-entendus. Les propositions pouvaient aussi être exprimées dans un face à face direct, incitant l'étudiante à se rapprocher ou lui offrant de venir rencontrer l'homme dans sa chambre et, dans quelques cas, ces avances sont allées jusqu'à s'inviter directement.

Il y a eu le guide aussi. II m'a payé une bière. Je la prends tranquillement puis il me dit : "Est-ce que nous allons dans ta chambre? »-"Non, je ne te connais pas. »- - Oui mais nous allons jaser. » J'ai dit non. Alors [plus tard], il est venu cogner à ma porte, il s'est installé sur mon lit et j'étais incapable de le faire partir. Il essayait de s'appuyer la tête sur ma cuisse. Je disais : «Non non, je ne veux pas, c'est quoi ton problème? » Il ne voulait pas sortir, jusqu'à ce que je sois tellement mal à l'aise. J'avais les larmes aux yeux. Là, il a compris que ça n'avait pas de bon sens. Je n'aurais pas dû lui ouvrir la porte, mais tu te dis: "J'ai confiance ». Nous en avons discuté avec les autres filles et nous nous sommes rendu compte qu'il avait essayé avec tout le monde.

Le refus d'implication des étudiantes pouvait aussi provoquer des réactions d'agressivité et de rancœur de la part des hommes qui ont été repoussés.

\section{Représentations du monde féminin}

Selon Medimegh-Dargouth (1992), la Tunisie est le seul pays arabe qui ait réussi, grâce aux réformes structurelles du Code du statut personnel, à remettre en question le statut de la femme dans la société musulmane traditionnelle, pour les intégrer à la sphère publique et les amener à participer plus pleinement au développement du pays. Cependant, la perception des étudiantes du monde féminin est pour le moins réduite et elles s'entendent pour dire que les femmes étaient peu présentes dans les lieux publics et que leurs contacts avec elles étaient plutôt rares. Bien que plusieurs aient rencontré, lors du congrès ou d'activités sociales parallèles, des femmes de milieux professionnels et établi avec elles des échanges jugés intéressants sur la culture et la société tunisiennes, d'autres ont été frappées par l'étroitesse d'esprit de certaines des intervenantes.

C'est par le biais des expériences du hammam (ou bain maure) qu'elles ont pu avoir un aperçu plus direct du monde féminin. Cette institution occupe une place importante pour les deux sexes, à la fois pour des raisons d'hygiène, de relaxation et de sociabilité poussée, de sensualité, sinon d'érotisme. Jusqu'à l'âge de quatre ans, quelquefois davantage, les garçons accompagnent leur mère et leurs sœurs au hammam, avant d'y devenir persona non grata. Couchard (1994) rapporte même à ce sujet que tout homme porte en lui le complexe du hammam, regrettant amèrement ce paradis perdu, un lieu de «fantasmatisation » poussée, comme le suggère le film de Ferid Boughedir, Halfaouine (1990). Dans une ambiance chaude, humide et détendue, à cause de la température du lieu et de son humidité élevée, les femmes se déplacent à leur aise et s'adonnent à des rituels de corps (frottage, gommage et massage du corps, shampoing et teinture, épilation du corps). Ces pratiques, qui commencent à un âge précoce, sont particulièrement importantes avant les fêtes ou le mariage quand la future mariée visite le hammam en compagnie des membres féminins de sa famille, de ses voisines et de ses amies, un rite accompagné de chants et d'amusements, pour être préparée à la cérémonie ultérieure. L'expérience du hammam par les étudiantes leur a permis de se sensibiliser aux caractéristiques du monde féminin tunisien plus privé et à la liberté qui y règne, contrairement aux comportements plus contraints observés dans la sphère publique.

On est allé dans un bain public et là on a pu parler aux femmes parce que c'est la seule place où elles se rencontrent juste entre elles; c'est la place pour parler parce qu'à part ça elles ne sont pas dans des endroits publics, elles restent à l'intérieur.

Par contre, plusieurs étudiantes disent ne pas s'être senties confortables dans ce contexte à cause du dévoilement total de leur nudité dans un contexte public amplifié par l'homosensualité qui s'y rattache et ce, malgré les marques de respect qui leur étaient prodiguées ; elles affirmaient avoir aussi pris conscience des différences fondamentales dans les règles culturelles associées à la pudeur.

Ça m'a vraiment impressionnée. Nous, les occidentales, on se promène à moitié nue dans la vie publique et dans la vie privée on a de la difficulté à ôter le petit restant. Pour elles, la sphère privée les protège, elles sont bien. C'est ce qui se dégageait d'elles : elles étaient bien dans leurs mouvements.

Parce que ce qui est drôle c'est que dans les rues, dans les endroits publics, les femmes sont cachées; elles ont l'air d'être assez pudiques, puis nous on est toutes dévoilées. Puis, au bain, c'est nous qui nous cachions, qui étions gênées alors qu'elles n'avaient pas de problèmes avec la nudité. Finalement c'est nous qui avions l'air le plus fou!

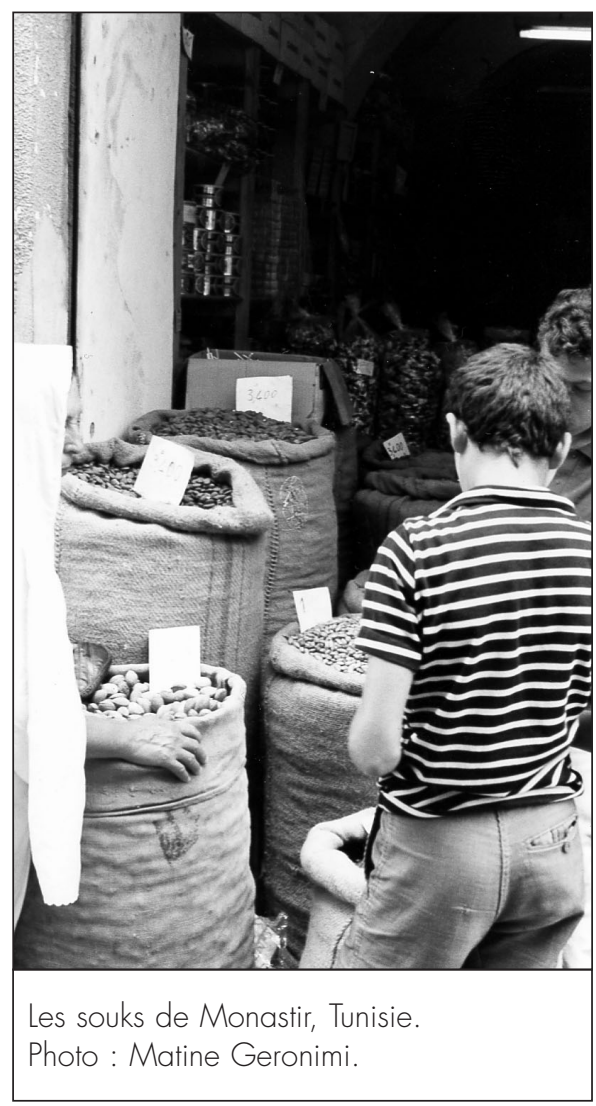




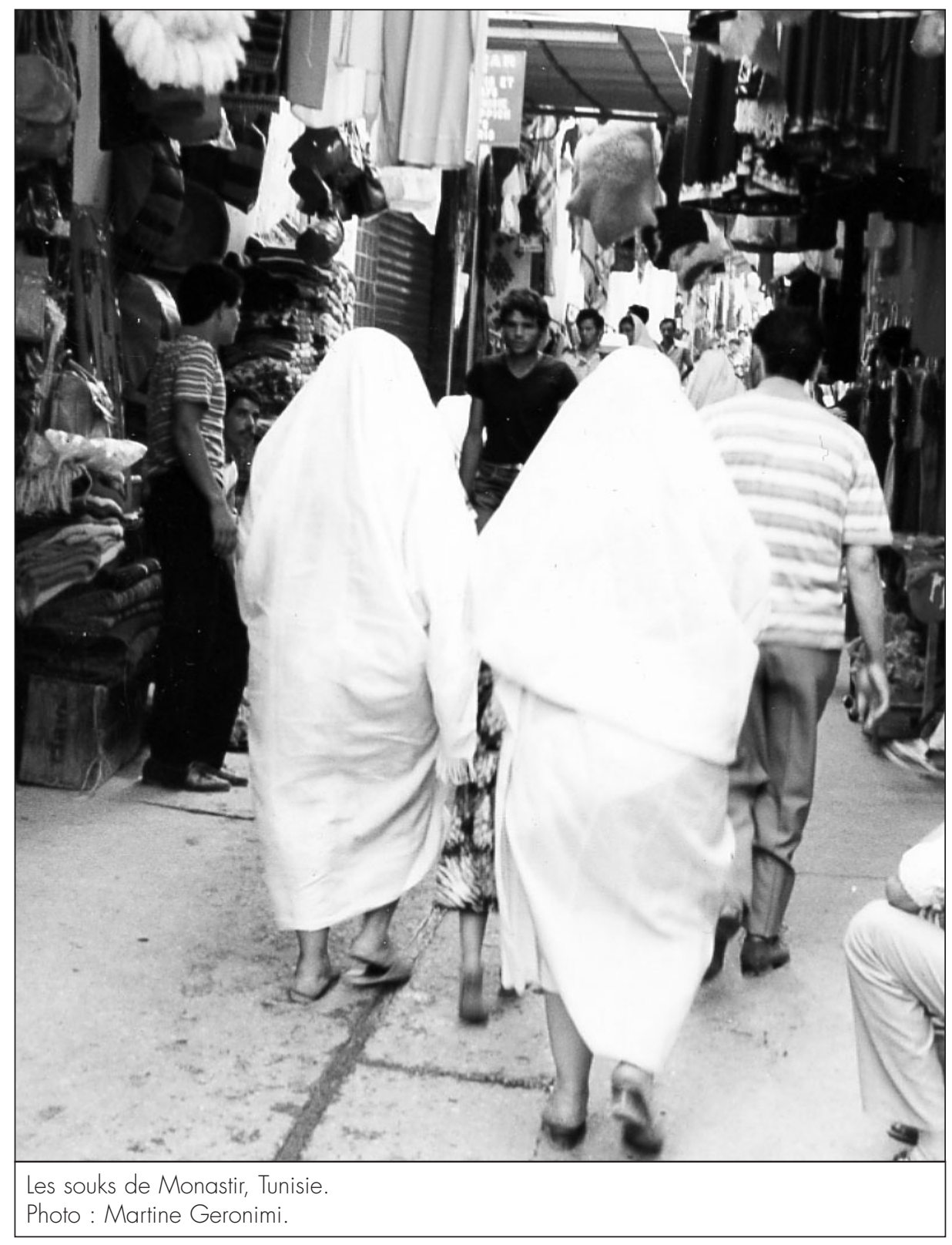

En plus, il fallait se déshabiller devant les autres filles du groupe, en plus des autres femmes tunisiennes présentes. Les Tunisiennes étaient très à l'aise dans leur nudité, contrairement à nous. Notre pudeur n'avait pas sa place au hammam où il n'y a pas de barrières. C'était paradoxal de voir leur ouverture au hammam, contrairement à la rue, et l'opposé pour nous.

\section{Retour sur le voyage}

Le retour sur le voyage a donné lieu à plusieurs commentaires. Pour plusieurs, le ryth-
Un drôle de retour, j'ai été malade et j'avais comme un "écoeurement » du voyage. Était-ce dû au virus ou était-ce autre chose? Je ne le sais pas. Je n'avais pas envie d'en parler pour l'instant, j'avais peu envie de mûrir mes pensées, mes sentiments, pour y voir plus clair, car il s'est passé beaucoup de choses en peu de temps. Je retournerais un jour en Tunisie, mais peut-être dans un autre contexte, car ce n'était pas vraiment des vacances. Le rythme du voyage était accéléré, stressant et je suis revenue brûlée.

Plusieurs ont noté des déficiences dans l'organisation du congrès, mais ont apprécié l'occasion d'explorer le pays, d'en découvrir les paysages, surtout ceux du Sud, et de se confronter à un dépaysement profond. Si certaines ont davantage retenu les expériences déplaisantes, surtout avec les hommes par lesquels elles se sont senties agressées et harcelées, d'autres se sont senties sevrées de la convivialité et de la chaleur des relations avec les Tunisiens, qu'elles ont contrastées avec celles des milieux nord-américain et québécois.

En revenant, je m'ennuyais un peu des contacts humains ; là-bas, les gens sont chaleureux, accueillants et aimaient te recevoir chez eux. ils prennent soin de toi. Ici, c'est plus snob.

Ici, en Amérique du Nord, nous avons des préjugés énormes et ça nous limite dans nos expériences. Je veux voyager toute ma vie et je veux retourner là-bas, car j'ai aimé ça.

Ce rythme accéléré et les chocs culturels qui l'ont accompagné ont provoqué, chez plusieurs, une fatigue profonde accompagnée de symptômes physiques et psychiques.

Le lendemain [de mon retour], j'ai été malade, j'étais faible. Je ne voulais rien voir de ce qui s'appelait la Tunisie. J'étais vraiment épuisée du voyage parce qu'on ne s'est vraiment pas reposé beaucoup là-bas. Après, quand j'ai repris mes énergies, j'ai pu montrer mes photos et là je considère que j'ai fait un beau voyage. Cela a pris une période d'adaptation.

\section{Conclusion}

Les expériences de voyage d'étudiantes québécoises en Tunisie, telles que rapportées lors d'entrevues, mettent en relief les caractéristiques des chocs culturels qu'elles ont expérimentés. Ces caractéristiques ont moins porté sur le climat, la géographie ou les problèmes de langue que sur la sphère de la qualité des interactions avec les hommes et les femmes et les différences d'ethos associées aux contrastes dans la construction du rapport au regard et aux 
contacts physiques, de même qu'aux variations dans les codes de séduction et de pudeur. Ainsi, les modes de communication non verbale liés au regard et au toucher qui sont plus dominants dans l'aire arabo-musulmane semblent être perçus par les étudiantes comme des formes de harcèlement, sinon d'agression, ce qui confirme les hypothèses de Watson (1970), de Watson et Graves (1966) et d'Argyle et Dean (1965). La méconnaissance de la culture locale, en favorisant le maintien d'habitudes vestimentaires qui n'obéissent pas aux canons de modestie en vigueur en Tunisie, a pu aussi contribuer à amplifier les approches de séduction ou des comportements perçus comme du harcèlement et provoquer, en retour, des réactions d'anxiété et d'énervement chez les touristes.

Les étudiantes ont par ailleurs expérimenté une vaste gamme de relations avec les hommes intéressés à développer des relations amicales, romantiques ou plus ouvertement sexuelles et provenant en particulier des «dragueurs » ou du personnel associé au monde touristique. Ces données suggèrent que les modèles d'interaction avec la population locale demandent à être étudiés dans un cadre plus large que celui défini par l'opposition entre «tourisme sexuel » et «tourisme romantique » (Sanchez-Taylor, 2001) et à inclure d'autres modalités.

En outre, cette étude révèle particulièrement l'importance d'étudier les modalités de contacts des femmes avec d'autres femmes qui peuvent révéler des contrastes importants dans le rapport aux vêtements, au corps et à son dévoilement. À cet égard, l'expérience du hammam est significative des oppositions dans les règles d'expression de la pudeur dans les sphères publique et privée et met en relief les modalités contrastées d'inscription des femmes dans les contextes socio-sexuels québécois et tunisien. D'autres études dans cette perspective devraient être entreprises afin de mieux cerner la richesse et les variations dans le vécu touristique des femmes et les contraster avec celles des hommes, pour ainsi mieux comprendre comment le genre intervient dans la construction des expériences de voyage.
Słéphanie Laporte est détentrice d'un baccalauréat en sexologie de l'Université du Québec à Montréal et poursuit actuellement une maîtrise en sexologie (option counselling). Elle travaille comme assistante de recherche sur le projet d'étude de la cohorte Oméga portant sur les HARSAH.

\section{Bibliographie}

" La Tunisie, nouvelle destination d'hiver », La Presse, Cahier H, p. 1-2, 14 décembre 2002.

Argyle, Michael, et Janet Dean (1965), « EyeContact, Distance and Affiliation », Sociometry, vol. 28, p. 289-304.

Boughedir, Ferid (1990), Halfaouine, Film franco-tunisien.

Chebel, Malek (1995), Encyclopédie de l'amour en Islam, Paris, Payot et Rivages.

Couchard, Françoise (1994), Le fantasme de séduction dans la culture musulmane, Paris, Presses universitaires de France.

De Albuquerque, Karl (1998), « Sex, Beach Boys, and Female Tourists in the Caribbean ", Sexuality and Culture, vol. 2, p. 87-111.

De Kadt, Emanuel J. (1979), Tourisme : passeport pour le développement?, Paris, Economica.

Goffman, Irving (1966), Behavior in Public Places: Notes on the Social Organization of Gatherings, New York, Free Press.

Herold, Edward S., Rafael Garcia, et Tony DeMoya (2001), «Female Tourists in the Dominican Republic: Romance or Sex Tourism? », Annals of Tourism Research, vol. 28, p. $978-997$.

Johnston, L. (2001), «(Other) Bodies and Tourism Studies », Annals of Tourism Research, vol. 28, p. 180-201.

Karch, Dieter, et G. Dann (1981), « Close Encounters of the Third World ", Human Relations, vol. 34, p. 249-268.

Lévy, Joseph J., Stéphanie Laporte, et Mansour El Feki (2001), " Tourisme et sexualité en Tunisie », Anthropologie et Sociétés, vol. 25, p. 61-68.

Maison de l'islam (2003), « Quelles limites à l'action du regard ? Pourquoi ?» [http:// www.maison-islam.com/article.php?sid=20].

Maurer, Mechtild (1992), Tourisme, prostitution, SIDA, Paris, L'Harmattan.

McGehee, Nancy G., Lorie Loker-Murphy, et Muzaffer Uysal (1996), «The Australian
International Pleasure Travel Market: Motivations from a Gendered Perspective », The Journal of Tourism Studies, vol. 7, p. $45-57$.

Medimegh-Dargouth, Aziza (1992), Droits et vécu de la femme en Tunisie, Paris, Éditions L'Hermès-Edilis.

Meish, Lynn A. (1995), "Gringas and Otavalenos: Changing Tourist Relations », Annals of Tourism Research, vol. 22, p. 441462.

Netzley, Patricia D. (2001), Encyclopedia of Women's Travel and Exploration, Oryx Press.

Pruitt, Deborah, et Suzanne Lafont (1995), « For Love and Money: Romance Tourism in Jamaica », Annals of Tourism Research, vol. 22, p. 422-440.

Sanchez-Taylor, Jacqueline (2001), « Dollars Are a Girl's Best Friend? Female Tourists Sexual Behaviour in the Caribbean ", Sociology, vol. 35, p. 749-764.

Schmitt, Arnos, et Jehoda Sofer (1992), Sexuality and Eroticism among Males in Moslem Societies, New York, Editions Haworth.

Smaoui, Ahmed (1992), « Le développement du tourisme en Tunisie et le rôle des organisateurs de voyages étrangers ", dans Jean-Louis Michaud (dir.), Tourismes Chance pour l'économie, risque pour les sociétés ?, Paris, Presses Universitaires de France, p. 127-150.

Watson, Michael O. (1970), Proxemic Behavior: A Cross-Cultural Study, Paris, The Hague, Mouton.

Watson, Michael O., et Theodore D. Graves (1966), « Quantitative Research in Proxemic Behavior », American Anthropologist, vol. 68, p. 971-985. 